\title{
珠芽蓼种群克隆多样性及克隆结构的初步研究
}

\author{
陆建英 ${ }^{1}$ 马瑞君 ${ }^{2 *}$ 孙 坤 $^{1}$ \\ (1 西北师范大学生命科学学院, 兰州 730070) (2 韩山师范学院生物系, 广东潮州 521041)
}

\begin{abstract}
摘 要 珠芽苶 (Polygonum viviparum) 是青藏高原东缘广泛分布的克隆植物, 具有有性和无性 (根状茎和珠芽)两种 生殖方式。该研究采用 RAPD 技术对分布于不同海拔的珠芽苶 7 个自然种群进行了克隆结构和克隆多样性(是单 克隆种群还是多克隆种群) 以及克隆多样性与海拔因子之间的相关性研究, 为了解高山克隆植物对环境的适应性 策略及揭示克隆植物的繁殖和分布特点提供科学依据。研究结果表明 :1) 采用 13 条 RAPD 引物对珠芽苶 7 个种群 共 140 个样本进行扩增分析，共扩增到 117 个位点, 其中多态性位点 84 个, 多态位点百分率 PPL 达到 $71.79 \%$ 检测 到 43 个基因型, 且全部为局限型基因型,2)与 Ellstrand 和 Roose (1987)总结的克隆植物的克隆多样性平均值相比 $(P D=0.17, D=0.62)$ 珠芽苶种群克隆多样性水平稍高 Simpson 指数平均为 0.639 基因型比率 $P D$ 平均为 0.307 ; 3) 克隆结构分析表明, 珠芽蓼种群内克隆之间的镶嵌明显, 这可能与珠芽蓼过渡型的克隆构型有关。研究中珠芽 蓼种群的构型有游击型、密集型以及这两者之间的过渡类型; ;)采用 SPSS 软件对珠芽苶种群的克隆多样性与海拔 高度进行相关性分析 结果显示它们之间并无明显的相关性。
\end{abstract}

关键词 珠芽苶 克隆植物 克隆多样性 克隆结构 RAPD

\section{CLONAL DIVERSITY AND STRUCTURE IN POLYGONUM VIVIPARUM}

\author{
LU Jian-Ying ${ }^{1}$, MA Rui-Jun ${ }^{2 *}$, and SUN Kun ${ }^{1}$ \\ ${ }^{1}$ College of Life Science, Northwest Normal University, Lanzhou 730070 , China, and ${ }^{2}$ Biology Department, Hanshan Normal University, \\ Chaozhou , Guangdong 521041 , China
}

\begin{abstract}
Aims Polygonum viviparum, which is widely distributed in eastern Qinghai-Tibet Plateau in China , is a clonal plant species with two modes of reproduction , clonal propagation by below-ground rhizomes and bulbils and sexual by seeds. This study investigated clonal structure and diversity (monoclonal or multiclonal population) and the relationship between clonal diversity and the altitude. Our objective was to examine the adaptive strategy of alpine plants to varied environments and establish a foundation for understanding reproduction and distribution of clonal plants.

Methods We selected seven populations in eastern Qinghai-Tibet Plateau along an altitudinal gradient and sampled 20 individuals in each population with at least $1 \mathrm{~m}$ between individuals to avoid individuals from the same clone. We employed random amplified polymorphic DNA (RAPD) technology for studying clonal structure and diversity and analyzed the relationship between clonal diversity and altitude by SPSS software.

Important findings We selected 13 random primers for amplification and found 117 repetitive loci with 84 polymorphic loci (total average percentage of polymorphic loci was $71.79 \%$ ). We differentiated 43 RAPD genotypes among the 140 plants sampled. Mean Simpson's index was 0.639, and mean PD was 0.307, slightly higher than the mean of Ellstrand $(P D=0.17, D=0.62)$. There was a clear mosaic among $P$. viviparum clones, probably because of diverse clonal structure. The formations of $P$. viviparum populations included guerilla, phalanx and a transitional type. Clonal diversity was independent of altitude.
\end{abstract}

Key words Polygonum viviparum, clonal plant, clonal diversity, clonal structure, RAPD

许多植物，特别是生长在逆境中的植物，常具有 性生殖和克隆繁殖的两种增加个体数量的方式。其 中克隆繁殖具有可塑性强、繁殖速度快、幼苗成活率 高可在短期内占据不同生境,尤其是有适应不良环
境的优势 (Barret \& Shore,1989)，对物种的繁衍具有 重要意义。因此，生长于干旱、高山等胁迫环境下的 植物, 其有性生殖方式往往被限制, 而克隆繁殖占优 势 ( Hartmann ,1957 ; Gray ,1993 ;Stöcklin \& Bäumler， 
1996 ;Klimes et al. ,1997)。植物种群克隆结构及克 隆多样性是种群进化过程中适应环境的结果, 深入 研究种群的克隆结构及克隆多样性是了解克隆植物 种群的形成、维持和衰退机制的重要方面, 同时对研 究植物定居、侵殖和演替的机理也有重要意义(阮成 江等 ,2005)。近年来, 对克隆植物的克隆结构、克隆 多样性和克隆大小的研究已成为克隆植物的研究热 点之一(Sipes \& Wolf ,1997 ;Suyama et al. 2000)。由 于克隆繁殖后代具有与母体植物相同的遗传特性 （阮成江等，2005）,克隆繁殖具有适应胁迫环境方面 的优势，因此，前人的研究表明，随着海拔的升高，传 粉昆虫数量减少, 植物花期缩短, 以及小种群效应产 生, 高山克隆植物克隆繁殖也会增加, 克隆多样性水 平下降 (Younga et al.,2002) ; 但 Stenstorm 等(2001) 则认为虽然海拔与温度呈负相关性, 但海拔越高的 地方 植物的种子容易逃离动物的采食, 因此在海拔 较高处植物的遗传变异反而更丰富。可见，在环境 胁迫强度与克隆结构、克隆多样性之间的关系尚不 明确。

珠芽蓼 (Polygonum viviparum) 为蓼科蓼属的多 年生草本, 广布于亚洲、欧洲及美洲, 在我国常见于 陕西、甘肃、西藏、青海等地, 主要生长在海拔 $2200 \sim 4200 \mathrm{~m}$ 的高山草原湿润地区, 是优良的牧 草。珠芽蓼是典型的兼性克隆植物, 有性生殖为虫 媒传粉, 同时靠地下根状茎和珠芽行克隆繁殖(李有 忠和沈颂东, 1996)。本研究在青藏高原东缘沿海拔 梯度选择了 7 个珠芽蓼自然种群, 采用 RAPD 的方 法对其克隆结构、克隆多样性 (是单克隆种群还是多 克隆种群) 以及克隆多样性与海拔因子之间的相关 性进行了初步的研究, 旨在探讨高山克隆植物对环 境的适应性策略, 并为揭示克隆植物的繁殖策略、克 隆多样性与海拔之间的关系提供依据。

\section{1 材料和方法}

\section{1 实验材料}

实验材料采集于青藏高原东缘甘南藏族自治州 7 个不同海拔下的珠芽蓼自然种群 (图 1) , 海拔在 $2000 \sim 3900 \mathrm{~m}$ 之间不等, 采集时间在植物生长季 节(8月)，具体材料采集地的海拔及其生境见表 1 。 当地年平均降水量为 $620 \mathrm{~mm}$,多集中在 5 9 月, 年 平均气温为 $1.8{ }^{\circ} \mathrm{C}$ 。具体采样方法由于材料个体分 布所限，且尽可能避免采集到相同克隆，个体之间至 少相隔 $1 \mathrm{~m}$ 。采集珠芽蓼新鲜的嫩叶片后立即使用 变色硅胶干燥保存,带回备用。

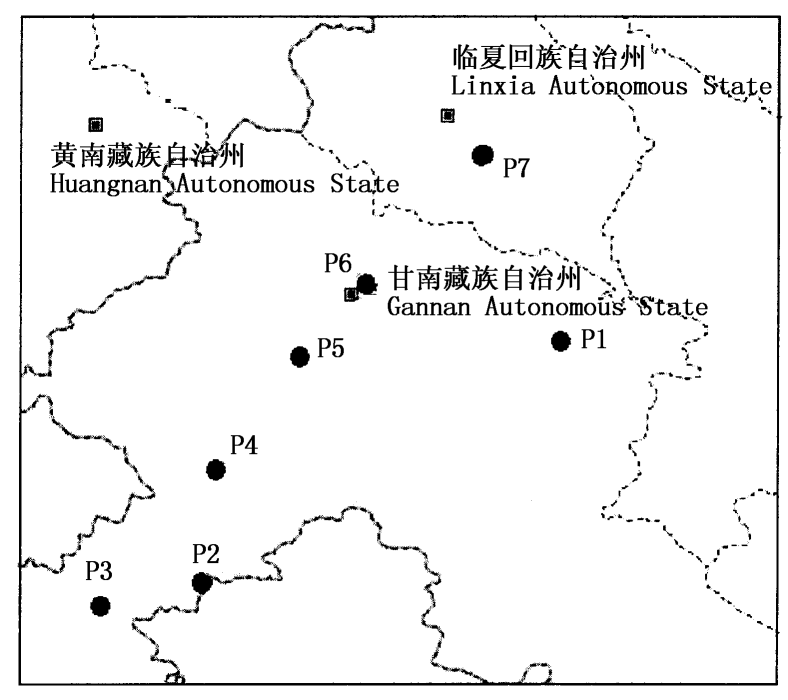

表示珠芽蓼 7 个种群的采样地点

Showing sampling places of seven populations of Polygonum viviparum 图 1 珠芽蓼种群采样分布图

Fig.1 The distribution map of study population of Polygonum viviparum

P1 : 治力关-临潭 Yeliguan-Lintan P2 : 尔玛梁 Gamaliang P3 :玛 曲 Maqu P4 : 尔海-碌曲 Gahai-Luqu P5 : 碌曲-合作 Luqu-Hezuo P6 : 依毛梁 Yimaoliang P7 : 和政南阳山 Hezheng Nanyangshan Mountain

表 1 珠芽蓼天然种群 RAPD 分析的样品及其生境条件

Table 1 The habitats in sampling sites of Polygonum viviparum

\begin{tabular}{cccc}
\hline $\begin{array}{c}\text { 种群 } \\
\text { Population }\end{array}$ & $\begin{array}{c}\text { 海拔 } \\
\text { Altitude }(\mathrm{m})\end{array}$ & $\begin{array}{c}\text { 采样个数 } \\
\text { No. of sample }\end{array}$ & $\begin{array}{c}\text { 生境 } \\
\text { Habitats }\end{array}$ \\
\hline P1 & 2500 & 25 & 山上 On the Mountain \\
P2 & 3900 & 25 & 路边草地 Roodside meadow \\
P3 & 3400 & 25 & 草地 Meadow \\
P4 & 3150 & 20 & 草地 Meadow \\
P5 & 3000 & 27 & 湿地 March \\
P6 & 2900 & 24 & 湿地 March \\
P7 & 2000 & 25 & 河滩 Beach \\
\hline
\end{tabular}

P1 P7 : 见图 1 See Fig. 1

\section{2 总 DNA 的提取与检测}

采用 CTAB 法(Doyle \& Doyle ,1987) 提取基因组 总 DNA, 方法略有改进。温育后将离心速度由 100 $g$ 提高到 $10000 \mathrm{~g}$, 提高第二次抽提时加入抽提液 的量由原来的 $800 \mathrm{ul}$ 变为 $900 \mathrm{ul}$,并且 $10000 \mathrm{~g}$ 离 心。加入异丙醇后, 放到常温下沉淀 $30 \mathrm{~min}$ 。提取 后用紫外分光光度计检测 DNA 模板的浓度, 用 $0.8 \%$ 的琼脂糖凝胶电泳、EB 染色、紫外灯下观察照 相, 电泳结果 DNA 条带清晰、无拖尾现象表明 DNA 质量较好。最后将样品稀释到 $5 \sim 10 \mathrm{ng} \mathrm{ul}^{-1}$ 备用。

1.3 引物的筛选和 PCR 扩增及产物

引物篮选时采用两轮篮选, 首先用每个种群的 2 个样品对 200 条引物 (上海生工公司) 进行第一轮 
篮选，篮选出扩增片段清晰、特异性好的引物 82 条， 然后用这 82 条引物对每个种群的 4 个样品进行扩 增,篮选出扩增带型稳定、重复性好的 13 条引物作 为全部样品扩增引物, 表 2 为引物的序列及其检测 到的位点数。选取每个种群的 20 个样品进行 PCR 扩增。采用 $10 \mu \mathrm{l}$ 的反应体系: 模板 $5 \sim 10 \mathrm{ng} \mathrm{ul}^{-1}$, 50 mmol L ${ }^{-1}$ Tris-HCl (pH 8.3) 500 ug ul-1 BSA ,Ficoll $10 \%$,dNTP 各 $200 \mathrm{umol} \mathrm{L}^{-1}$,Taq 酶 $0.5 \mathrm{U}$,引物 $3 \mathrm{ng} \mathrm{ul}^{-1}, \mathrm{MgCl}_{2} 2 \mathrm{mmol} \mathrm{L}^{-1}$, 酒石黄 $1 \mathrm{mmol} \mathrm{L}^{-1}$ 。 PCR 反应在德国 Biometra UNO II PCR 仪上进行,扩 增程序为: 94 ${ }^{\circ} \mathrm{C}$ 预变性 $5 \mathrm{~min} ; 94{ }^{\circ} \mathrm{C} \quad 1 \mathrm{~min}, 37{ }^{\circ} \mathrm{C}$ $1 \mathrm{~min}, 72{ }^{\circ} \mathrm{C} 1 \mathrm{~min}, 40$ 个循环,72 ${ }^{\circ} \mathrm{C}$ 延伸 7 min。在 含有 $\mathrm{EB}$ 的 $1.5 \%$ 的琼脂糖凝胶上电泳 $2 \sim 3 \mathrm{~h}$, 然后 在凝胶成像系统上观察比较并照相。

表 2 用于珠芽蓼扩增的随机引物的序列及其检测的位点数

Table 2 The sequence of primers and the number of tested loci

\begin{tabular}{lccc}
\hline $\begin{array}{c}\text { 引物 } \\
\text { Primer }\end{array}$ & $\begin{array}{c}\text { 序列 }\left(5^{\prime}-3^{\prime}\right) \\
\text { Sequence }\left(5^{\prime}-3^{\prime}\right)\end{array}$ & $\begin{array}{c}\text { DNA 总位点数 } \\
\text { Total number of loci }\end{array}$ & $\begin{array}{c}\text { 多态位点数 No. of } \\
\text { polymorphic loci }\end{array}$ \\
\hline S5 & TGCGCCCTTC & 10 & 7 \\
S8 & GTCCACACGG & 13 & 7 \\
S12 & CCTTGACGCA & 7 & 6 \\
S13 & TTCCCCCGCT & 8 & 6 \\
S223 & CTCCCTGCAA & 7 & 4 \\
S393 & ACCGCCTGCT & 8 & 5 \\
S494 & GGACGCTTCA & 10 & 6 \\
S500 & TCGCCCAGTC & 11 & 7 \\
S1102 & ACTTGACGGG & 10 & 8 \\
S1107 & AACCGCGGCA & 7 & 6 \\
S1156 & CACAACGGGA & 10 & 9 \\
S1420 & CTTCTCGGAC & 8 & 7 \\
S2113 & CCGCCGGTAA & 8 & 6 \\
\hline
\end{tabular}

\section{4 数据分析}

扩增结果用 $\lambda$ DNA100 bp 做分子标记，对照反应 产物在胶上的对应位置，同一位点的产物按有带的 计为 1 ,无带的计为 0 ,形成二元数据矩阵。分析中 将在各扩增位点的 RAPD 谱带完全相同的个体视为 同一克隆(即基因型相同)。为便于和其它植物进行 比较, 我们计算了反映克隆种群的克隆多样性及其 空间结构的几种常用度量指标。这些指标的意义及 其计算方法详见有关文献 ( Nei ,1973;Pielou ,1969； Fager, 1972;Eckert \& Barrett ,1993)。为了了解不同克 隆之间的遗传关系, 在 DCFA1.1 (张富民和葛颂， 2002)中由 RAPD 数据得到不同克隆的欧氏距离平 方, 通过 NTSYS2.02 的 SHAN 程序聚类得到聚类图。 采用 SPSS12.0.1 软件进行克隆多样性与海拔高度
相关性的分析。

\section{克隆多样性分析指标：}

1)种群中基株数目 $(G)$ : 将全部位点基因型相 同的植株视为来自同一基株, $G$ 即为种群中基株总 数。

2)平均克隆大小 $(N / G)$ : 即每个基株平均含有 的克隆分株数, $N$ 是样本大小。

3 )不同基因型比率 $(P D)$ : 以 $G / N$ 估算,$N$ 是样 本大小。

4) Simpson 多样性指数 ( D ) : Simpson 指数最初是 用于测定种的多样性和均匀度的 现在用于度量种 群内的克隆多样性 (Parker \& Hamrick,1992)。公式 为:

$$
D=1-\frac{\sum\left(N_{i}\left(N_{i}-1\right)\right)}{N(N-1)}
$$

式中, $N_{j}$ 为第 $j$ 个基因型的分株数, $N$ 是样本大小。

5) Fager 指数 $(E)$ (Fager, 1972) :

$E=\left(D-D_{\min }\right) /\left(D_{\max }-D_{\min }\right) ; D_{\min }=(G-1)$

$(2 N-G) / N(N-1) ; D_{\max }=(G-1) N / G(N-1)$

6）局限型基因型 :只在一个种群中出现的基因 型(Elstrand \& Roose ,1987)。

7) 广布型基因型 :在 75\%以上种群中出现的基 因型(Elstrand \& Roose ,1987)。

\section{2 结果与分析}

\section{1 珠芽苶种群的 RAPD 扩增与克隆多样性}

从 200 个 RAPD 引物中篮选出 13 个引物对珠 芽苶 7 个种群的 140 个 DNA 样品进行 PCR 扩增, 其 中引物 S12 对 P4 种群的扩增见图 2 (其它扩增图 略)。13 个引物共检测到 117 个位点, 其中多态性 位点 84 个,多态位点百分率 PPL 达到 $71.79 \%$ 。

表3是各研究种群的平均克隆大小、基因型比

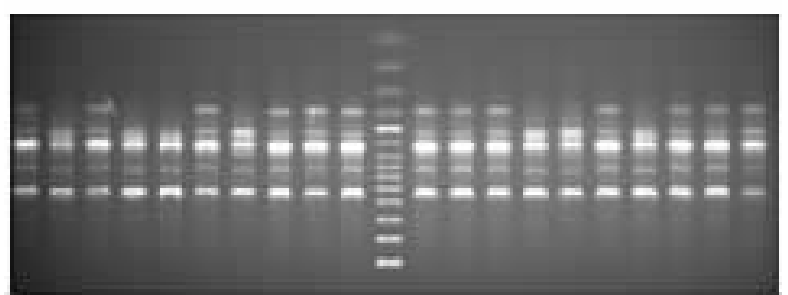

$12815673310 \div 1113: 511: 5161$ 1 141020

图 2 引物 S12 扩增的 P4 种群的 DNA 片段图谱

Fig. 2 The PCR amplification pattern of P4 population generated with primer S12

1 20 :P4 种群的 20 个样品 20 samples of P4 population $\mathrm{M}$ 分子 量标准 (100 bp DNA ladder plus) Standard marker 
率、Simpson 多样性指数等统计值。珠芽苶种群平均 基株数目为 6 个;平均克隆大小为 3.622 ,从 2.000 到 5.000 不等 ,P3 和 P6 两个种群最高, 都为 5.000 , $\mathrm{P} 1$ 种群最低为 2.000 ;基因型比率平均为 0.307 (0.200 0.500) ;由 Simpson 多样性指数反映出的克 隆多样性显示, P1 种群最高 $(0.879), \mathrm{P} 6$ 最低 (0.284) 平均为 0.639 。珠芽苶种群 Fager 指数的平
均值只有 0.495 , 低于 Ellstrand 和 Roose(1987) 对于 21 种克隆植物的比较总结结果 $(E=0.68)$, 其中 P6 种群的均匀度 $E$ 为 0 , 说明在这个种群内 1 种基因 型占据了绝大多数个体。如表 4 ,珠芽蓼克隆繁殖 的 7 个珠芽蓼种群都是由多基因型构成, 基因型个 数在各种群中分布不均种群间基因型多样性较低, 7 个种群共 140 个样本 检测到 43 个基因型(克隆)。

表 3 珠芽蓼种群的克隆多样性

Table 3 Clonal diversity in all populations

\begin{tabular}{|c|c|c|c|c|c|c|}
\hline $\begin{array}{c}\text { 种群 } \\
\text { Population }\end{array}$ & $\begin{array}{c}\text { 样本大小 }(N) \\
\text { Sample size }\end{array}$ & $\begin{array}{l}\text { 种群基株 }(G) \\
\text { Number of genets }\end{array}$ & $\begin{array}{c}\text { 平均克隆大 }(N / G) \\
\text { Average size of genotype }\end{array}$ & $\begin{array}{c}\text { 基因型比率 }(P D) \\
\text { Proportion of distinct } \\
\text { genotypes }\end{array}$ & $\begin{array}{c}\text { Simpson 多样性指数 }(D) \\
\text { Simpson diversity index }\end{array}$ & $\begin{array}{c}\text { 均匀度 }(E) \\
\text { Fager's evenness }\end{array}$ \\
\hline $\mathrm{P} 1$ & 20 & 10 & 2.000 & 0.500 & 0.879 & 0.712 \\
\hline $\mathrm{P} 2$ & 20 & 8 & 2.500 & 0.400 & 0.816 & 0.684 \\
\hline P3 & 20 & 4 & 5.000 & 0.200 & 0.500 & 0.427 \\
\hline P4 & 20 & 5 & 4.000 & 0.250 & 0.800 & 0.911 \\
\hline P5 & 20 & 7 & 2.857 & 0.350 & 0.689 & 0.441 \\
\hline P6 & 20 & 4 & 5.000 & 0.200 & 0.284 & 0.000 \\
\hline P7 & 20 & 5 & 4.000 & 0.250 & 0.505 & 0.288 \\
\hline 平均 Mean & 20 & 6 & 3.622 & 0.307 & 0.639 & 0.495 \\
\hline
\end{tabular}

表 4 种群内克隆数目和构型分布

Table 4 Clones number and clonal architecture within populations

\begin{tabular}{|c|c|c|c|}
\hline 种群 & 克隆数 & 构型 & 种群内克隆分布情况(不同克隆的植株用 / 隔开) \\
\hline Population & Number of clones & Clonal architecture & Clones distribution within population (different clones distingwished by 'l') \\
\hline P1 & 10 & 过渡型 Transition & $12,19 / 18 / 20$ \\
\hline $\mathrm{P} 2$ & 8 & 过渡型 Transition & 13,20 \\
\hline P3 & 4 & 密集型 Phalanx & 14/13/18-20 \\
\hline P4 & 5 & 游击型 Guerilla & 13 15/8-10/18-20 \\
\hline P5 & 7 & 游击型 Guerilla & 19/13/17, 14, 20/5/8/11, 18, 16, 15, 12, 10, 9, 7, 6, 4, 1 \\
\hline P6 & 4 & 密集型 Phalanx & 20/5/16/18, 19, 17, 6-15, \\
\hline P7 & 5 & 密集型 Phalanx & 1/2-4, 6-15, 17/5/16/18-20 \\
\hline
\end{tabular}

\section{2 珠芽蓼的克隆结构}

表 4 是通过 RAPD 方法计算分析出的珠芽苶 7 个种群的克隆分布情况。7 个种群的克隆数目 (基 株数目) 大小不同 (4 10 个)。所有的种群都是多 克隆种群, 没有发现有单克隆种群。从种群内克隆 的空间分布看 相邻个体属于同一克隆的可能性较 大，但也有相距较远的个体具有相同的基因型，例如 P2、P4 和 P5 种群的部分个体, 这表明珠芽苶种群内 克隆有明显的镶嵌现象。同时,通过 Excel 软件分 析得出了珠芽蓼种群内的克隆基株的频率分布图 (图 3), 可以看出,P3、P6 种群的基株数目最少 (4 个) 其中 P3-1 型的克隆占了 P3 种群的 70\% ,P6-1 型的克隆占了 P6 种群的 $85 \%$,成为各自种群内具
有竞争优势的克隆;P7 种群内的基株数目也较少， 有 5 个, 且其中 P7-2 基因型的个体占了 $70 \%$,成为 P7 种群内优势克隆。

\section{3 克隆种群的聚类分析}

通过 Ntsys 软件对珠芽蓼种群个体进行聚类分 析结果表明珠芽苶同一种群的个体先聚为一类， 再与其它种群个体聚类, 只有少数个体先与其它种 群个体聚在了一起。图 4 为珠芽苶 P3 种群的聚类 结果(其它种群聚类图略)。可以看出,P3 种群基本 聚为 4 支, 其中个体 $1 \sim 11 、 15 \sim 17$ 属于同一克隆, 个体 12、14 属于同一克隆, 18 20 属于同一克隆, 只 有个体 13 是一个单独的克隆，它的基因型和其它任 何个体都不相同, 但遗传距离与 P3-11 克隆十分接 
近, 很有可能是由 P3-11 克隆突变形成的。

\section{4 珠芽苶的克隆多样性与海拔的关系}

采用 SPSS 软件对珠芽苶种群的克隆多样性与 海拔高度进行相关性分析，结果显示，Simpson 多样 性指数和海拔相关不显著, 相关系数 $r=0.223$ 。这 表明 珠芽苶种群的克隆多样性和海拔梯度不存在 明显的相关性。
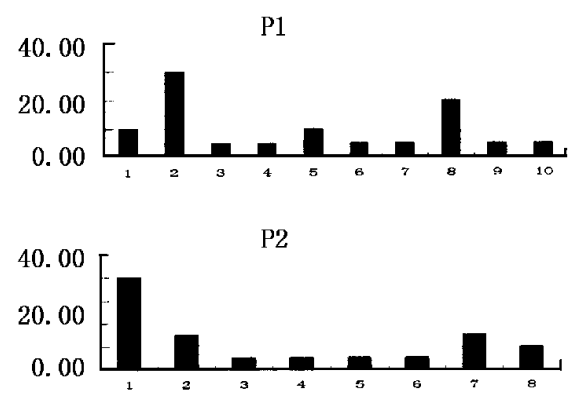

P3

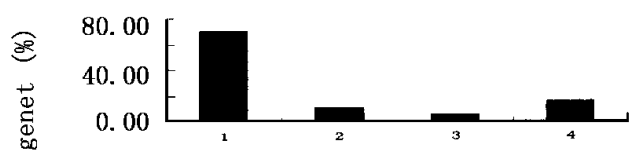

P4

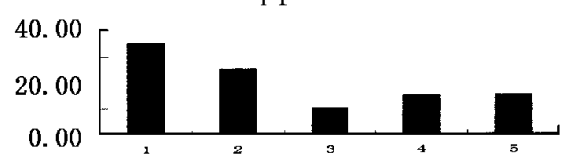

P5

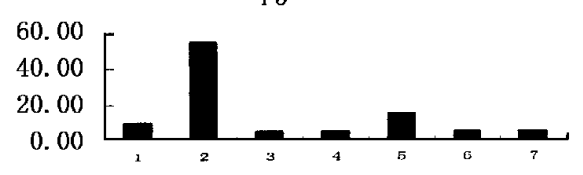

P6

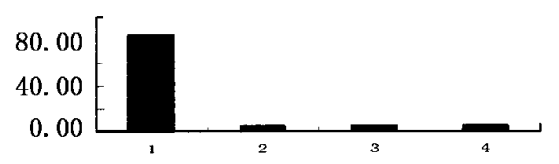

P7

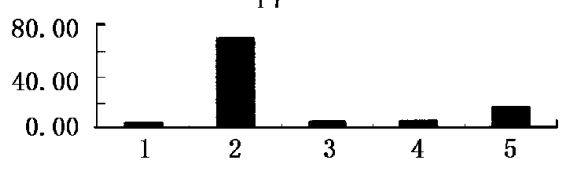

基株数目 Number of different genet

图 3 各种群内克隆基株的频率分布

Fig.3 Frequency distribution of genets within each population

\section{3 讨 论}

\section{1 珠芽苶种群的克隆多样性}

本实验研究结果显示, 珠芽苶种群的不同基因 型比率平均为 0.307 ,Simpson 多样性指数平均为 0.639 和其它多克隆种群的平均值 $(P D=0.17, D=$ $0.62)$ (Ellstrand \& Roose ,1987)相比稍高。这就说明
青藏高原东缘分布的珠芽苶种群克隆多样性较高。 另外, Bauert( 1993) 用琼脂糖凝胶电脉的方法检测了 珠芽蓼 4 个种群的遗传变异性，在其中也发现了 15 种不同的基因型。本研究中珠芽蓼克隆多样性的维 持, 主要有几个方面：一方面可能是因为地处青藏高 原东缘的珠芽苶种群在定居初期或发展过程中, 恶 劣的高山环境使得种群为了适应环境压力, 偶尔进 行种苗补充 增加种群竞争能力; 另一方面, 微环境 的异质性会通过多样性选择而促使不同的基因型的 新个体占据不同的微环境。另外, 体细胞突变也是 其遗传变异的一个持续来源。Lynch 和 Milligan (1994)和 Gill 等 (1995)认为在克隆植物中以体细胞 突变的高发生率补偿有性繁殖重组的缺失, 从而使 克隆繁殖种适应环境的变化。

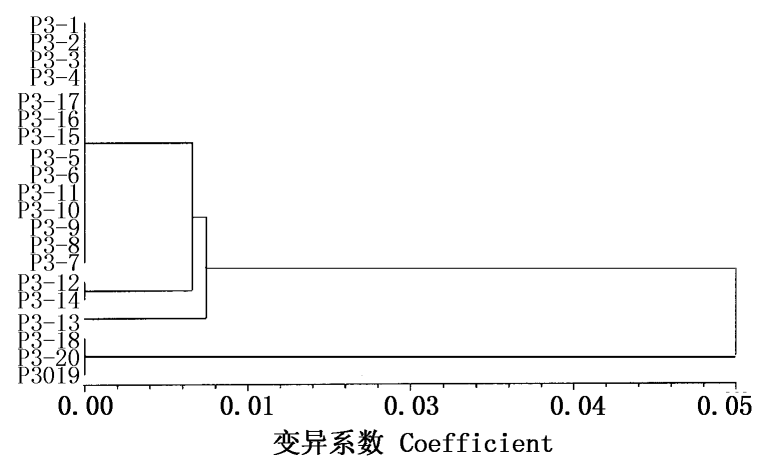

图 4 珠芽蓼 P3 种群的 UPGMA 法聚类分析图 Fig.4 Dendrogram of UPGMA cluster analysis of Polygonum viviparum $\mathrm{P} 3$ population

相关性分析结果表明, 珠芽苶种群的克隆多样 性和海拔梯度不存在明显的相关性, 即随着海拔的 升高，珠芽蓼并未在有性生殖与克隆繁殖之间进行 明确的权衡, 这一结果与前人的研究均不一致 (Younga et al. 2002 :Stenstorm et al. 2001)。究其原 因可能有:1)在研究地的海拔范围内 海拔高度的变 化还不足以影响传粉昆虫的数量、植物花期与种子 传播等与有性繁殖密切相关的因素。因此, 珠芽苶 的有性生殖和克隆繁殖的相对比重并未发生明显的 改变 或者其改变尚不足以影响克隆多样性水平 2) 珠芽蓼种群分布于较高的海拔条件下，经过长期的 自然选择 其在垂直分布上有着广泛的生态适应性 ; 3) 克隆植物在不同的环境中可能面临着不同的选择 压力 环境异质性能促进植物不同基因型的固定 (Hangelbroek et al.,2002 ;Torimaru et al. ,2003)。这 就说明影响植物克隆多样性的因素不仅仅是海拔梯 度, 而是由环境异质性和不同地区的物候条件等各 
个因子共同作用的结果。此外, 也有可能受到其它 非生态因子，如人为、交通工具或动物取食强度等的 影响。

\section{2 珠芽蓼种群的克隆结构}

克隆分株种群的空间格局被认为是克隆植物与 其利用环境 (如资源) 异质性能力相关的性状: 游击 型构型具有很强的克隆扩散能力,易于利用分散分 布的资源滵集型构型的克隆扩散能力有限, 易于利 用非常局部分布的资源(陈锦华等 2003)。

本研究中通过 RAPD 数据研究分析得到珠芽苶 种群内的克隆个体在空间上的分布情况。比较珠芽 蓼自然种群可以看出, 7 个克隆种群均属于多克隆 种群, 并不是单一的或只有几个克隆组成的种群。 不同种群包括的克隆数目不同, 有的种群含有 10 个, 有的却只有 4 个, 这可能是由不同海拔条件下种 群环境的异质性决定的。珠芽蓼种群内不同克隆间 相互交错混生, 克隆之间的镶嵌明显, 这与它的克隆 构型密切有关。从种群内克隆的空间分布情况看， 珠芽苶克隆种群的构型为混合型, 其中不同的种群 有着不同的构型, 包括游击型、密集型以及这两者之 间的过渡类型。这种混合型的克隆生长空间格局形 成了基株之间的镶嵌分布, 使得克隆植物具有最大 限度的利用资源的能力, 在生存竞争中能使种群得 以保存和发展。

克隆植物中不同基因型的适合度和竞争能力是 克隆植物研究中的重要问题, 但有关的研究还很少 (Ellstrand \& Roose ,1987;Montalvo et al . 1997)。从各 种群内克隆基株的频率分布图可以看出, 尽管珠芽 蓼不同种群中平均克隆所含分株数相差很大 (每个 基株平均有 $2 \sim 5$ 个分株), 但是在多数种群内都有 一到几个基因型占据相当大的比例，这说明不同的 克隆 (基因型) 可能具有不同的生长潜力或适合度， 进而可能具有不同的竞争能力, 而那些不适应于生 长环境的克隆个体(基因型)势必随着时间的推移最 终被淘汰, 这就是自然选择的结果。当然, 本研究只 是对珠芽苶的克隆结构和克隆多样性进行了初步研 究, 且因 RAPD 方法鉴定克隆与引物的多少密切相 关, 有一定的局限性。因此, 今后还可采用其它分子 标记 (AFLP、SSR、ISSR) 对珠芽苶的克隆规模大小、 克隆间的竞争及繁殖方式权衡等方面作进一步研 究。

\section{参 考 文 献}

Barret SCH, Shore JS (1989). Isozyme variation in colonizing plants. In: Soltis DE, Soltis PS eds. Isozymes in Plant Biology. Chapman \& Hall, Landon, 106 - 126.

Bauert MR (1993). Vivipary in Polygonum viviparum: an adaption to cold climate? Nordic Journal of Botany, 13, $473-480$.

Chen JH (陈锦华), Wang XF (汪小凡), Lü YT (吕应堂) (2003) . Pattern of clonal growth in a natural population of Sagittaria pygmaea Miq. (Alismataceae). Journal of Wuhan University (Natural Science Edition) (武汉大学学报 (理学版)), 49, 523 - 527. (in Chinese with English abstract)

Doyle JJ, Doyle JL (1987). A rapid DNA isolation procedure for small quantities of fresh leaf tissue. Phytochemistry Bulletin, 9, $11-15$.

Eckert CG, Barrett SCH (1993). Clonal reproduction and pattern of genotypic diversity in Decodon verticillatus (Lythraceae). American Journal of Botany, 80, 1175 - 1182 .

Ellstrand NC, Roose ML (1987). Patterns of genotypic diversity in clonal plant species. American Journal of Botany, 74, 123 131 .

Fager EW (1972). Diversity: a sampling study. The American Nature, $106,293-310$.

Gill DE, Lin C, Perkins SL (1995). Genetic mosaicism in plants and animals. Annual Review of Ecology and Systematics, 26, $423-444$.

Gray A ( 1993 ). The vascular plant pioneers of primary successions: persistence and phenotypic plasticity. In: Miles J, Walton D eds. Primary Succession on Land. Blackwell, Oxford, UK, $179-191$.

Hangelbroek HH, Ouborg NJ, Santamaría L, Schwenk K (2002) . Clonal diversity and structure within a population of the pondweed Potamogeton pectinatus foraged by Bewick's swans. Molecular Ecology, 12, $2137-2150$.

Hartmann H (1957). Studien über die vegetative Fortpflanzung in den Hochalpen. Jahresbericht der Naturforschenden Gesellschaft Graubündens, 86, 3-168.

Klimes L, Klimesova J, Hendriks R, van Groenendael J (1997). Clonal plant architecture: a comparative analysis of form and function. In: de Kroon H, van Groenendael J eds. The Ecology and Evolution of Clonal Plants. Backhuys Publishers, Leiden, the Netherlands, $1-29$.

Li YZ (李有忠), Shen SD (沈颂东) (1996). A preliminary morphological and anatomical investigation in the vegetative and reproductive organs of Polygonum viviparum. Journal of Qinghai Normal University (Natural Science Edition) (青海师范大学学 报(自然科学版) ), 1, 34-40. (in Chinese)

Lynch M, Milligan BG (1994). Analysis of population genetic structure with RAPD markers. Molecular Ecology, 3, 91 - 99.

Montalvo AM, Conard SG, Conkle MT, Hodgskiss PD (1997). Population structure, genetic diversity, and clone formation in Quercus chrysolepis (Fagaceae). American Journal of Botany, $84,1553-1564$.

Nei M (1973). Analysis of gene diversity in subdivided popula- 
tions. Proceedings of the National Academy of Sciences of the United States of American, 70, 3321 - 3323.

Parker KC, Hamrick JL (1992) . Genetic diversity and clonal structure in a columnar cactus, Lophocereus schottii. American Journal of Botany, 79, $86-96$.

Pielou EC (1969). An Introduction to Mathematical Ecology. Wiley-Interscience, New York.

Ruan CJ (阮成江), He ZX (何祯祥), Zhou CF (周长芳) (2005). Plant Molecular Ecology (植物分子生态学). Chemical Industry Press, Beijing. (in Chinese)

Sipes SD, Wolf PG (1997). Clonal structure and patterns of allozyme diversity in the rare endemic Cycladenia humilis var. jonessii (Apocynaceae). American Journal of Botany, 84, $401-409$.

Stenstrom A, Jonsson BO, Jonsdottir IS, Fagerstrom T, Augner M (2001). Genetic variation and clonal diversity in four clonal sedges ( Carex) along the Arctic coast of Eurasia. Molecular Ecology, 10, 497 - 513 .

Stöcklin J, Bäumler E (1996) . Seed rain, seedling establishment, and clonal growth strategies on a glacier foreland. Journal of Vegetation Science, 7, 45-56.

Suyama Y, Obayashi K, Hayashi I (2000). Clonal structure in a dwarf bamboo (Sasasenanesis) population inferred from amplified fragment length polymorphism (AFLP) fingerprints. Molecular Ecology, 9, 901 - 906 .

Torimaru T, Tomaru N, Nishimura N, Yamamoto S (2003). Clonal diversity and genetic differentiation in Ilex leucoclada M. patches in an old-growth beech forest. Molecular Ecology, 12, 809 818 .

Younga AG, Hill JH, Murray BG, Peakall R (2002). Breeding system, genetic diversity and clonal structure in the subalpine forb Rutidosis leiolepis F. Muell. (Asteraceae). Biological Conservation, 106, $71-78$.

Zhang FM (张富民), Ge S (葛颂) (2002). Data analysis in population genetics. I . Analysis of RAPD data with AMOVA. Biodiversity Science (生物多样性)，10,438 - 444. (in Chinese with English abstract) 